WellBeing International

WBI Studies Repository

1996

\title{
Quantity-Based Interference and Symbolic Representations in Chimpanzees (Pan troglodytes)
}

S. T. Boysen

The Ohio State University

G. G. Berntson

Emory University

M. B. Hannan

The Ohio State University

J. T. Cacioppo

Emory University

Follow this and additional works at: https://www.wellbeingintlstudiesrepository.org/acwp_asie

Part of the Animal Studies Commons, Comparative Psychology Commons, and the Other Animal Sciences Commons

\section{Recommended Citation}

Boysen, S. T., Berntson, G. G., Hannan, M. B., \& Cacioppo, J. T. (1996). Quantity-based interference and symbolic representations in chimpanzees (Pan troglodytes). Journal of Experimental Psychology: Animal Behavior Processes, 22(1), 76.

This material is brought to you for free and open access by WellBeing International. It has been accepted for inclusion by an authorized administrator of the WBI Studies Repository. For more information, please contact wbisr-info@wellbeingintl.org.

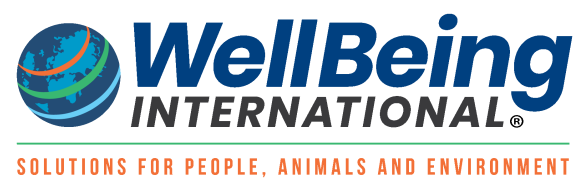




\title{
Quantity-Based Interference and Symbolic Representations in Chimpanzees (Pan troglodytes)
}

\author{
S.T. Boysen ${ }^{1,2}$, G.G. Berntson ${ }^{1,2}$, M.B. Hannan ${ }^{1}$, J.T. Cacioppo ${ }^{1}$ \\ ${ }^{1}$ Ohio State University \\ ${ }^{2}$ Emory University
}

\begin{abstract}
$\underline{\text { ABSTRACT }}$
Five chimpanzees with training in counting and numerical skills selected between 2 arrays of different amounts of candy or 2 Arabic numerals. A reversed reinforcement contingency was in effect, in which the selected array was removed and the subject received the nonselected candies (or the number of candies represented by the nonselected Arabic numeral). Animals were unable to maximize reward by selecting the smaller array when candies were used as array elements. When Arabic numerals were substituted for the candy arrays, all animals showed an immediate shift to a more optimal response strategy of selecting the smaller numeral, thereby receiving the larger reward. Results suggest that a response disposition to the high-incentive candy stimuli introduced a powerful interference effect on performance, which was effectively overridden by the use of symbolic representations.
\end{abstract}

There is little question that chimpanzees represent an extant species with cognitive capabilities often reminiscent of human reasoning abilities and conceptual skills (e.g., Boysen, Berntson, Shreyer, \& Hannan, 1995; Fujita \& Matsuzawa, 1986; Gillan, Premack, \& Woodruff, 1981; Matsuzawa, 1985; Premack, 1986; Savage-Rumbaugh, 1986). Over the past two decades, numerous investigations with captive chimpanzees have focused on demonstrations of such skills, with either the overt or veiled suggestion that these abilities are analogous to similar capacities or cognitive processes in humans. In most cases, however, elucidation of the underlying cognitive mechanisms or the processes by which abilities were acquired have not been forthcoming. If not cautious, one can be left with the impression that chimpanzees are fundamentally equivalent to humans in most cognitive domains. It is perhaps the nature of the scientific enterprise, however, that when an animal fails to learn a task or acquire some specific skill, reports typically do not make their way into the archival literature. Nevertheless, the failure to acquire a particular task or to demonstrate some ability can sometimes be more compelling and informative than success, especially if the determinants and mechanisms of that failure can be identified.

We recently encountered a rather striking training failure with 2 chimpanzees, in a study initially designed to explore strategic deception in this species. What was surprising was that the task was seemingly unremarkable, simply requiring a selection between two different-sized candy arrays (Boysen \& Berntson, 
1995). Two chimpanzees worked together, with one (the selector) given the opportunity to make choices between the candy arrays. A reversed reinforcement contingency was imposed, so that the candies in the selected array were given to a passive observer animal. The selector chimp then received the remaining, nonselected array. Thus, the function of the selector was to choose the array that the observer was to receive, with the selector then getting what was left over. In order to maximize payoff, it was in the best interest of the selector animal to choose the dish with the smaller number of candies, in order to reap the larger remainder. However, both animals in the preliminary study failed to do so, even after hundreds of training trials. Instead, they persisted in generally choosing the dish that contained the larger number of candies and, thus, consistently received the smaller amount of reward. These findings are reminiscent of the self-control literature in humans, in which children, for example, have difficulty inhibiting a direct response to food in order to obtain a larger, but delayed, reward (Mischel, Shoda, \& Rodriguez, 1989).

To further explore this curious performance failure, we capitalized on the previously acquired skills of one of the animals (Sheba) in counting and in the use of Arabic numerals as quantity symbols (Boysen, 1993; Boysen \& Berntson, 1989b). All of the task rules and features were identical, except that Arabic numerals, which represented the number of reinforcers (candies) that would be provided to the chimpanzees, were substituted for the candy arrays. Under these conditions, Sheba was able to immediately invoke the optimal food-sharing rules, which had been enforced since the start of the study, and now regularly received the larger candy array. As long as Arabic numerals served as stimuli, Sheba consistently selected the smaller numeral and earned the greater number of candies. When candies were reintroduced as stimuli, her performance immediately deteriorated but returned to more optimal levels when Arabic numerals were again substituted for the candy array stimuli (Boysen \& Berntson, 1995).

We suggested that there were two incompatible behavioral or evaluative dispositions operative in this task-one associative and the other probably nonassociative. An associative disposition to select the smaller of the two arrays would be expected to develop on the basis of the instrumental reward contingency. That such a disposition had in fact developed was apparent in the optimal performance with Arabic numerals, despite the fact that this implicit knowledge did not manifest with candy arrays as stimuli. We attributed the poor performance with candy arrays to an interfering nonassociative disposition to respond to the direct perceptual or incentive features, or both, of the larger candy array. In Miller's (1959) terminology, this would be an example of an approach-approach conflict. We will return to this issue in the discussion section, as a potentially important role for symbols and symbolic representations may lie in the resolution of conflicts among incompatible dispositions.

The notable and persistent performance failure of the chimpanzees on the candy selection task provoked a number of questions that we address in the present studies. We first examine the generality of this interference effect and its resolution with symbols, as we now have 5 chimpanzees who are accomplished with Arabic numerals. We also evaluate the potential importance of the social foodcompetition context that was inherent in the design of our prior study. Finally, we explore the potential contribution of the quantities and incentive qualities of the stimulus-array elements to the taskinterference effect.

\section{General Method}

\section{Subjects}

Five chimpanzees (Pan troglodytes) served as subjects for each of the three experiments. Animals included 2 females (ages 12 years and 35 years) and 3 males (ages 6 years, 14 years, and 14.5 years). All of the animals had extensive experience and training on a variety of cognitive and behavioral tasks, including numerical matching of arrays of discrete items (0-6), selection of an Arabic numeral to match a 
sample array, and selection of an array to match a sample Arabic numeral (e.g., Boysen, 1993; Boysen \& Berntson, 1989b; Boysen, Berntson, Shreyer, \& Hannan, 1995; Boysen, Berntson, Shreyer, \& Quigley, 1993). Although some animals had considerably more breadth of experience with counting and other number-related skills (e.g., Sheba, Darrell), all had the numerical repertoires and conceptual competence necessary for the present series of experiments.

\section{Procedures}

All of the subjects were tested individually in a home caging area that could be directly accessed from the chimpanzees' outside play area. The testing space was equipped with a large polycarbonate window and steel shelf outside the window, on which the stimulus items were displayed. The chimpanzees made their choices by pointing to items, with the experimenter positioned out of view of the animal but able to clearly see their selections. All of the subjects were free to exit the testing situation at any time but were readily compliant and fully participated in testing.

For each trial, two arrays of candy (chocolate-covered peanuts), two arrays of medium-sized rocks (approximately $2 \mathrm{~cm}$ in diameter), or two Arabic numeral symbols (black numerals on a white $7.5 \times 12 \mathrm{~cm}$ background) were placed in two separate dishes. Dishes were placed in front of the subject, approximately $30 \mathrm{~cm}$ apart, and the chimpanzee was permitted to select one of the two dishes (by pointing). Once a choice was made, the experimenter returned the contents of the selected dish to the candy, rock, or placard supply. The remaining candy array in the nonselected disk (or a number of candies corresponding to the nonselected Arabic numeral or rock array) was given to the chimpanzee. Thus, it was to the subject's advantage to select the dish containing the numerically smaller stimulus of the pair, in order to receive the larger number of candy reinforcers.

Test sessions were conducted twice daily, 5 days a week, between 9 a.m. and 4 p.m. The chimpanzees were maintained on their normal food ration throughout the study, which consisted of a variety of fresh fruits and vegetables given in feedings 3-5 times a day, with the final evening meal provided around 4:30 p.m. In addition, candy and other treats were used as reinforcers during testing on a variety of other cognitive tasks during the day, as well as rewards for compliant behavior (e.g., moving from one cage to another when requested by the experimenter). Although the animals were not food deprived, the candies were highly preferred items, and the animals were highly motivated to procure them.

\section{Statistical Analysis}

Primary statistical evaluation was by $t$ tests and regression analyses. For analysis of data from individual subjects, however, the nonparametric chi-square test for goodness of fit was used, as it does not assume independence of the measurements (Hays, 1988). Alpha level was set at .05 for all tests.

\section{Experiment 1}

The first experiment extended our finding of an interference effect in a quantity-judgment task to a larger group of animals. By testing animals in social isolation, we also examined the potential importance of the conspecific social context to the task-interference effect observed in our previous study (Boysen \& Berntson, 1995).

\section{Method}

Two quantities of candies (0-6 items) were placed in separate dishes (e.g., 2 candies in one dish, 5 candies in the other). The chimpanzees were permitted to choose one of the two dishes, and the contents of that dish were returned to the food supply in full view of the subject. The chimp was then given the 
candies from the remaining, nonselected dish. A total of 20 sessions were completed with each animal. Each session consisted of 20 trials (total of 400 trials per animal) with all possible combinations of the zero to six items, except one. In order to complete a trial block within each 20-trial session, one of the 21 possible number combinations was not tested ( 1 vs. 4 , selected randomly). In addition, one chimpanzee (Bobby) did not receive stimulus pairs with three or five items or the Arabic numerals 3 and 5 , as separate, ongoing experimental studies required that he not be exposed to these quantities or their associated numerals. Order of the number combinations within the sessions was randomized, and the left-right (L-R) position of each item was counterbalanced across trials.

\section{Results}

General performance. Overall results are illustrated in Figure 1. In accord with our preliminary study (Boysen \& Berntson, 1995), none of the 5 animals tested was able to reliably select the smaller of two candy arrays, despite the fact that this selection would yield the largest reward. As seen in Figure 1, performance was not random but was significantly below the expected chance performance of $0.50(M=$ $0.30, S E M=0.01), r(4)=27.47$. That is, the animals reliably selected the larger of the two candy arrays, even though this choice yielded the smaller amount of candy reward. There was no apparent improvement over the 20 sessions (400 trials), and performance over the last 10 sessions was virtually identical to that of the first 10 sessions $(0.29 \pm .01 \mathrm{vs}$. $0.31 \pm .02), t(4)=0.85, n s$. These results suggest that the incentive properties of the larger array may have introduced a selection bias that interfered with the optimal choice based on the extant reinforcement contingency (i.e., the selection of the smaller array in order to obtain the larger reward).

Array size and disparity. As illustrated in Figure 2A, performance on the task was highly related to the relative size disparity between the two choice arrays as well as to the overall array sizes. As might be expected, a greater degree of interference or bias against optimal performance was apparent on trials in which there was a larger disparity between the number of candies in the two arrays. The greater the size differential, the more likely that the animals would select the larger array and consequently receive a smaller reward. As shown in Figure 2A, however, this incentive disparity effect was qualified by the overall size of the arrays. Specifically, the interference effect for a given arithmetic disparity was diminished with larger mean array sizes. This suggests that the relevant dimension of disparity was not simply its absolute numerical value but the magnitude of the disparity with respect to the overall size of the reward. That is, the effects of a reward differential appeared to be described by a Weber-like function ( $\Delta$ reward/reward $=$ constant). This would predict that the behavioral effects of a given array combination would be linearly related not to the array disparity, per se, but rather to the ratio of the disparity to the overall array sizes.

This possibility was further examined by regression analyses. A disparity ratio was derived for each combination of paired arrays as the absolute numerical disparity between the arrays divided by the mean array size. For an overall analysis, mean performance (proportion of correct responses) across animals on each combination of paired arrays was then regressed against the disparity ratio for that pair of candy arrays. The results, illustrated in Figure $2 \mathrm{~B}$, revealed a highly significant relationship, with the disparity ratio accounting for approximately $93 \%$ of the variance in performance across all disparity ratios $\left(R^{2}=\right.$ $.93), F(1,18)=253.41$. As the disparity ratio increased, performance deteriorated. The slope of the function in Figure $2 \mathrm{~B}$ offers an index of the incentive processing of the stimuli, reflecting the extent of the task interference associated with incentive-disparity between the candy arrays. ${ }^{\dagger}$

Individual differences. There was notable consistency in performance across animals. This was especially notable, as 2 of the animals (Sheba and Sarah) had considerable previous experience in an essentially comparable task, whereas the others had not (see Boysen \& Berntson, 1995). The performance, as reflected in the overall proportion correct for each animal, was significantly below chance - all $\chi^{2} s(1, N=$ 
$400)=55.0-$ and fell within a narrow range of .27 to .31 . Similarly, there was little variation in the slopes of the disparity-ratio-performance functions across animals, as derived from separate within-subjects analyses $(M=-.53$, SEM $=0.02$; range $=.49$ to .58$)$. In each case, individual slopes accounted for an appreciable proportion of the performance variance for each individual subject (all $\left.R^{2} s>.70\right), F s(1,18)>$ 41.0.

Figure 1. Probability of optimal response of chimpanzees with candy array choice stimuli. Large (open) bar illustrates the mean performance across animals over all 20 sessions, and the heavy error bar illustrates the standard error of the overall mean. Smaller (cross-hatched) bars depict mean performance (and standard errors) within the 20 separate sessions. The dotted horizontal line depicts chance performance.

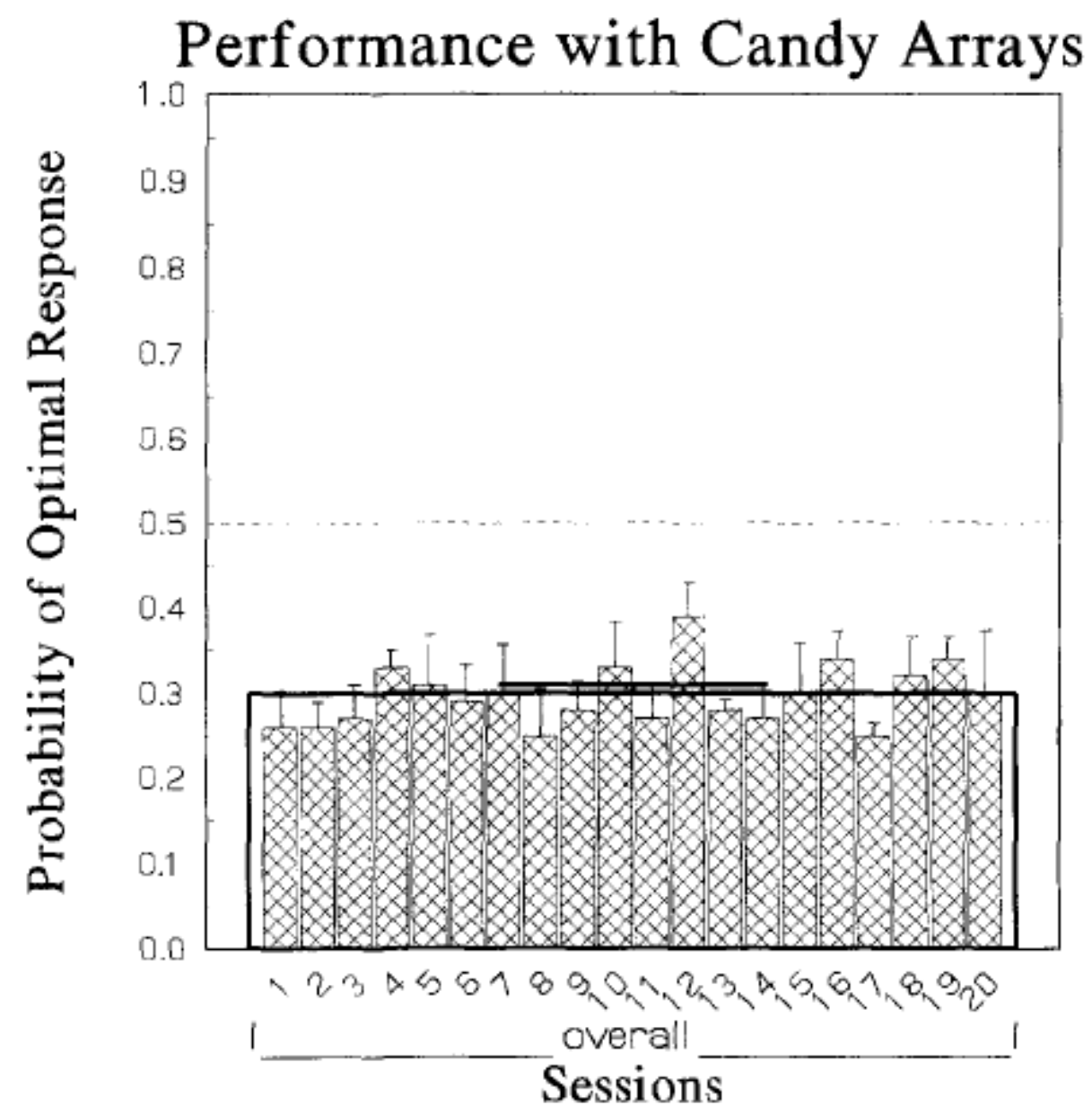

\section{Discussion}

In the present study, potential response biases arising from the immediate incentive features of the choice stimuli (i.e., the tendency to select the larger candy array) were pitted against the optimal response strategy (selection of the smaller array) based on the experimentally imposed reinforcement contingency. Results are consistent with our preliminary study (Boysen \& Berntson, 1995) and suggest that the incentive properties of the choice stimuli introduced a response bias that interfered with an optimal choice strategy based on the differential reward contingency. Our previous study (Boysen \& 
Berntson, 1995) entailed a social context in which an observer animal received the selected array and the subject, the nonselected array. The absence of the observer animal in the present study indicates that our prior results were not attributable to conspecific social competition. Indeed, there was substantial stability over time in the performance of the animal (Sheba), who had completed all phases of our previous study and was also tested in the present experiment. Despite the fact that the two studies were separated by almost 3 years, Sheba's overall performance in the comparable phase of the prior study (mixed arrays) was $34 \%$, compared with $27 \%$ in the present study.

Figure 2. Performance as a function of the numerical disparity among the paired stimuli and the mean array size. A. Best-fit (spline) three-dimensional response surface, illustrating the probability of optimal response as a function of both disparity and array size. Solid dots depict mean performance for each of the array combinations tested. B. Regression function illustrating the relationship between the probability of an optimal response and the disparity ratio (numeric disparity/total size of the arrays). The heavy line depicts the mean regression function, and the dotted lines illustrate individual regression functions for the 5 subjects. Dots depict the mean data points for each array combination tested.
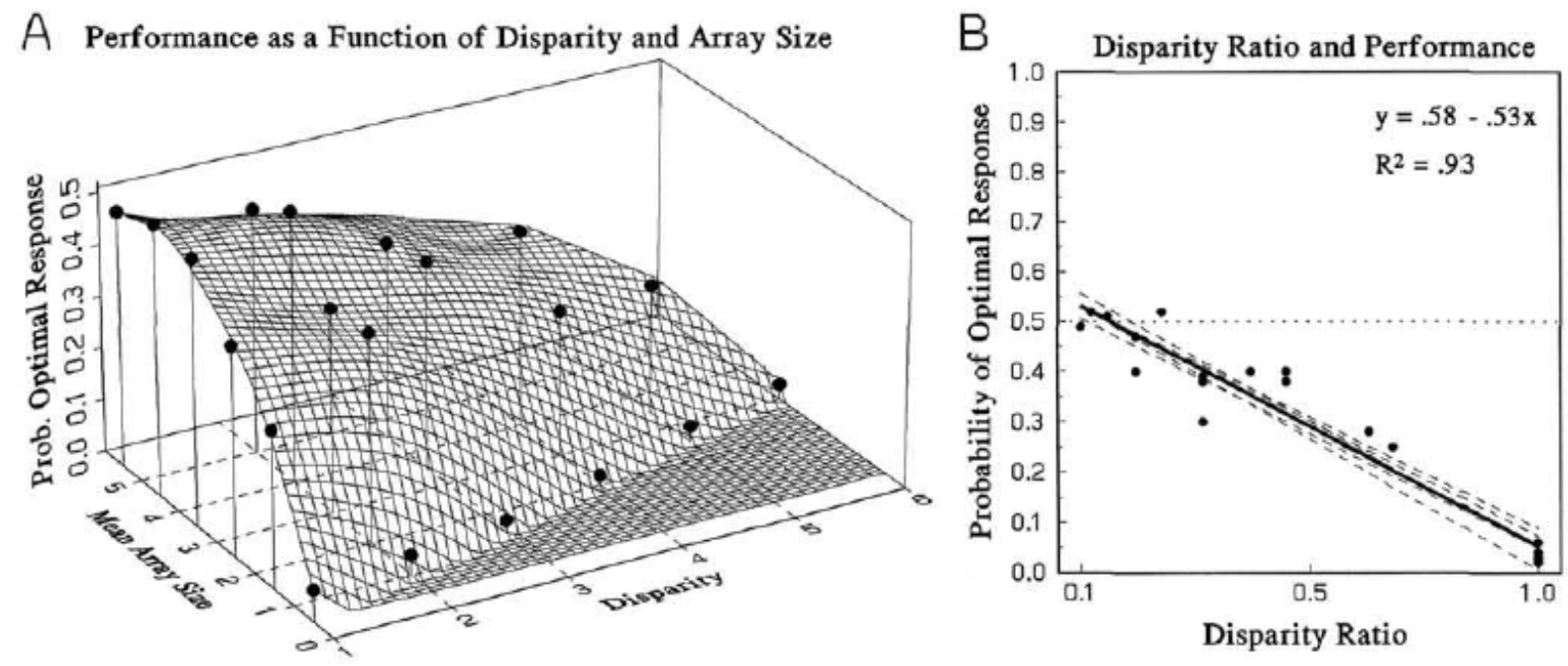

\section{Experiment 2}

In Experiment 1, response biases due to the incentive disparity between the choice stimuli yielded a powerful interference effect on optimal performance (as defined by the reinforcement contingency). It is not clear if this interference effect was dependent on the high incentive items (candies) that composed the stimulus-choice arrays or the perceptual features of the differing quantities that made up the arrays. This issue was addressed in Experiment 2, which was essentially comparable with Experiment 1, with the exception that the stimulus elements consisted of either candies or rocks in separate sessions.

\section{Method}

The testing context was identical to that of Experiment 1, with the exception that rock arrays, as well as candy arrays, were used as stimuli. ${ }^{\ddagger}$ Subjects were again required to choose between candy arrays or rock arrays (in different sessions) placed in two stimulus dishes. Once the animals made a choice between the two arrays, the selected candy or rock array was removed from the dish in full view of the subject and returned to supply bowls containing other candy or rocks. The subject then received the 
candy from the remaining, nonselected dish or a corresponding number of candies represented by the rocks in the nonselected dish. The array elements (candies or rocks) used were consistent within a session; arrays were composed either of all candies or all rocks.

All of the animals received an initial session using candies, followed by candy and rock sessions administered in counterbalanced order. Each animal was tested for a total of five sessions with candies and five with rocks. On the basis of the results of Experiment 1, a few changes were implemented in Experiment 2. A smaller number of sessions were given, in view of the session-to-session consistency obtained in Experiment 1. Consequently, a selected subset of stimulus combinations was used in order to increase the replicate trials with a given combination and still sample over a wide range of disparities and mean array sizes. Six stimulus combinations were used $(0 / 5,1 / 4,1 / 6,2 / 3,3 / 6,4 / 5)$, with each presented three times within a session. Trial order was randomized, and the L-R stimulus position was counterbalanced across trials. This yielded a total of 18 trials per session, for a total of 90 trials with each stimulus type (15 with each combination of array sizes).

Figure 3. Mean probability of optimal response of chimpanzees with candy arrays and rock arrays as choice stimuli. Large (open) bars illustrate overall mean performance of the animals across all sessions with each stimulus type. Smaller (cross-hatched) bars depict mean performance for the five separate sessions with each stimulus type. For illustration, session data are depicted in order within the stimulus type, but sessions with different stimulus types were tested in counterbalanced order. Error bars illustrate the standard errors. The dotted horizontal line depicts chance performance.

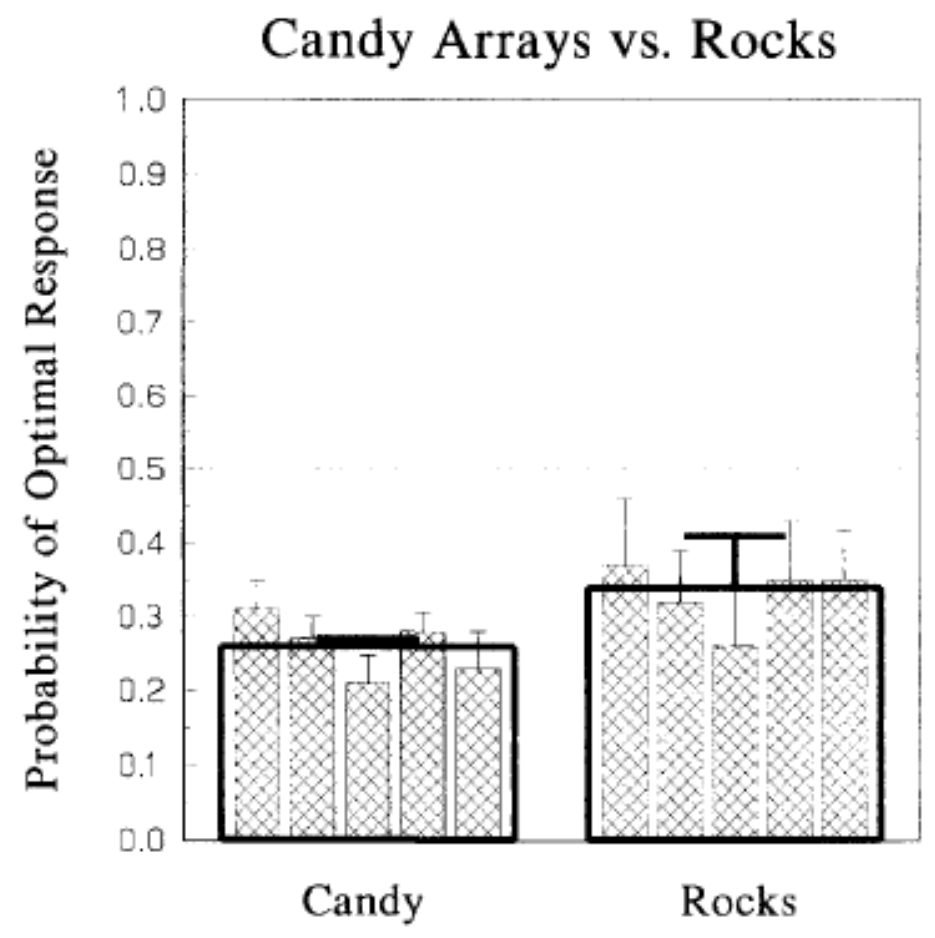

Results

General performance. Overall results are illustrated in Figure 3. Consistent with the findings from Experiment 1, animals were unable to reliably select the smaller of two candy arrays in order to obtain a 
larger reward. Performance with candy arrays was again significantly below chance $(M=0.26, S E M=$ $0.01), t(4)=16.51$, and no improvement in performance was apparent over that observed in Experiment 1. Rather, the animals continued to select the larger of the two candy arrays, thus receiving the smaller reward. Although their overall performance with rocks as array elements was also below chance $(M=$ $0.34, S E M=0.07$ ), it was not significantly so, $t(4)=2.49$. Performance with rocks also did not differ significantly from their performance with candies, $t(4)=0.92$, ns. These findings suggest that the interference effect arising from the numerical disparity between the stimulus arrays was not entirely dependent on the immediate, perceptual apprehension of high-incentive array elements (candies).

Figure 4. Regression functions illustrating the relationship between the probability of optimal responses and the disparity ratio (numeric disparityltotal size of the arrays) for candy and rock array stimuli. The heavy line depicts the mean regression function, and the dotted lines illustrate individual regression functions for the 5 subjects. Dots depict the mean data points for each array combination tested.
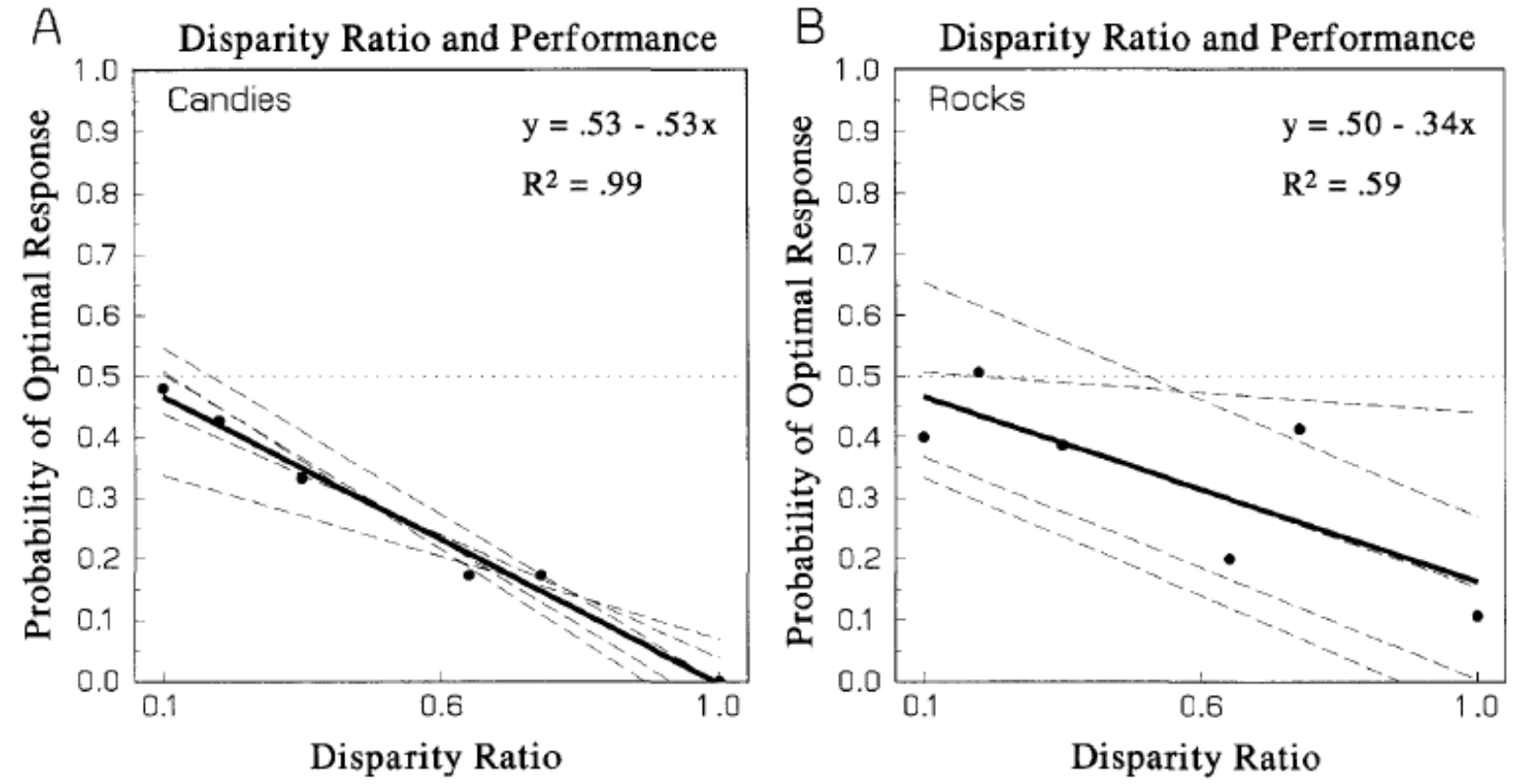

Array size and disparity. Consistent with the results of Experiment 1, performance with candy arrays was related to the disparity between the two arrays. A greater degree of interference was apparent on trials in which there was a larger disparity between the number of candies in the two arrays, and this effect was qualified by the mean size of the arrays. As illustrated in Figure 4A, the disparity ratio again accounted for the vast majority of the variance in overall performance across the different numerical combinations of paired arrays $\left(R^{2}=.99\right), F(1,4)=274.65$. The slope of the disparity-ratio function with candy arrays was identical to that obtained in Experiment 1 (see Figures $2 B$ and $4 A$ ). The results document a highly stable pattern of performance with candy arrays across both time and subjects.

As shown in Figure 4B, a generally similar pattern of results was apparent with rocks as array items. Although the mean slope with rocks was less steep than with candies, this difference was not significant (for candies, $M$ slope $=-.53, S E M=0.07$; for rocks, $M$ slope $=-.34, S E M=0.07$ ), $t(4)=2.22, n s$.

Individual differences. As in Experiment 1, there were minimal individual differences among the animals in their performance with candy arrays. Nonparametric analyses revealed each animal's performance was 
significantly below chance-all $X^{2} s(1, N=90)>12.8$-with all values falling within the narrow range of .22 to .31. Similarly, there was little variation in the slopes of the disparity-ratio functions across animals, as derived from separate within-subjects analyses $(M=-.53$, SEM $=0.02$; range $=.49$ to .58$)$. In each case, the individual slope accounted for an appreciable proportion of the performance variance of each subject across the differing array combinations $\left(R^{2} s=.51\right.$ to .90$)$.

Figure 5. Mean probability of optimal response of chimpanzees with candy arrays and Arabic numerals as choice stimuli. Large (open) bars illustrate overall mean performance of the animals across all sessions with each stimulus type. Smaller (crosshatched) bars depict mean performance for the six separate sessions with each stimulus type. For illustration, session data are depicted in order within the stimulus type, but sessions with different stimulus types were tested in counterbalanced order. Error bars illustrate the standard errors. The dotted horizontal line depicts chance performance.

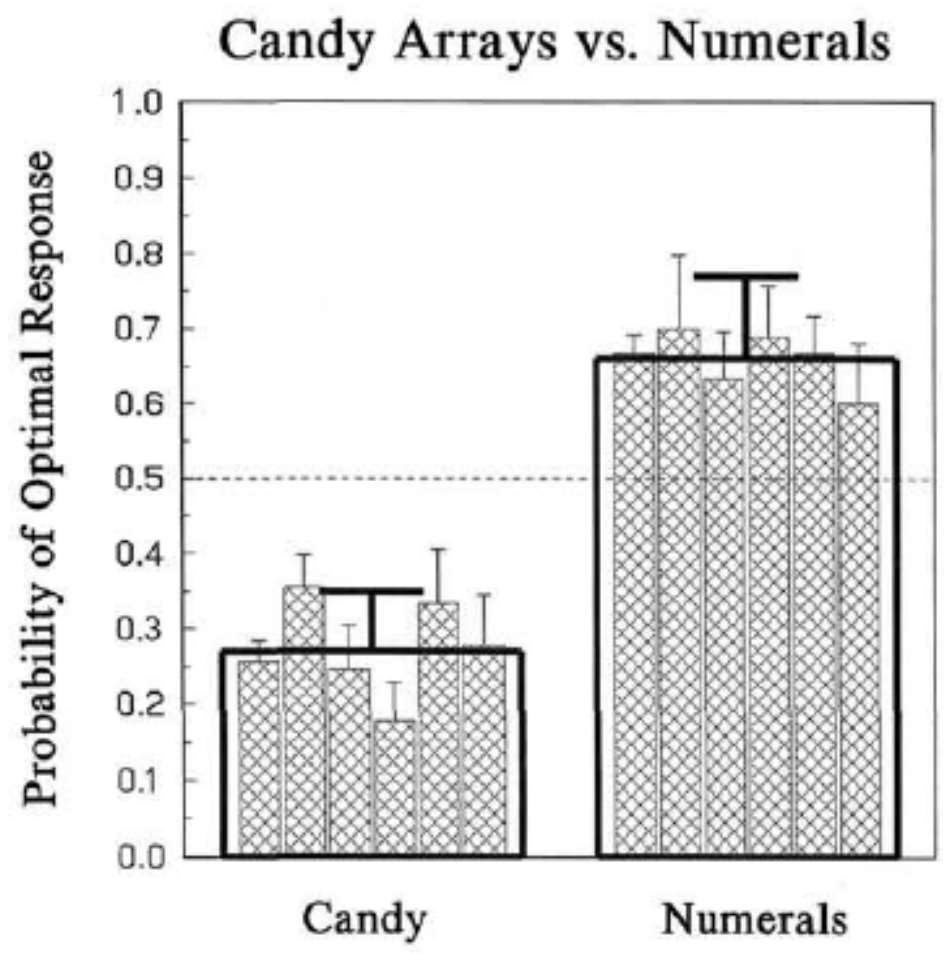

As illustrated in Figure 4, however, there was considerably greater animal-to-animal variation in performance with rocks as array elements. Three of the 5 animals displayed equivalent performance with candies and rocks, $X^{2} s(1, N=180)<2.2, n s$, although 2 subjects showed a significantly higher overall performance with rocks, $X^{2} s(1, N=180)>8.5$. This was associated with a decrease in the (negative) slope of the disparity-ratio function in one animal, suggesting diminished incentive dependent interference (Figure 4B). In contrast, improvement in the other animal was related to an overall increase in mean level of performance, without an appreciable slope change.

\section{Discussion}

The high-incentive items that composed the stimulus arrays of Experiment 1 likely contributed to the observed interference effect on performance. The persistence of interference with rock arrays in the present experiment, however, suggests that this interference may not be entirely accounted for by the 
inherent incentive properties of the array elements. Although it is unlikely that the rocks had any inherent incentive value for the animals, it is possible that they accrued incentive value through stimulus generalization or association with the candy reinforcers used throughout the experiment. We return to this issue below.

\section{Experiment 3}

Results of Experiment 2 suggest that the task-interference effect may be attributable in part to the incentive features of the differing quantities of items in the choice arrays. This was further examined in Experiment 3. On the basis of previous training with a variety of tasks, all of the animals had acquired facility with the use of Arabic numerals as symbolic representations for quantities (Boysen, 1993). Because Arabic numerals have neither inherent incentive value nor a perceptual similarity to the number of items they represent, number symbols provide a means to examine the contribution of the incentive features of the array stimuli to the interference effect observed in Experiments 1 and 2.

\section{Method}

The testing context and procedures were identical to that of Experiment 2, with the exception that candy arrays or Arabic numerals served as choice stimuli on separate sessions. Subjects were again required to choose between candy arrays and Arabic numerals placed in the two stimulus dishes, with the selected array returned to the supply bowls and the animal receiving the nonselected array (or the number of candies represented by the nonselected Arabic numeral). The type of stimulus (candies or numerals) was consistent within all trials of a given session.

All of the animals received an initial session using candies, followed by candy and Arabic numeral stimulus sessions administered in counterbalanced order. Each animal was tested for a total of six sessions with candies and six with rocks. The same six number combinations used in Experiment 2 were used in the present study, with trial order randomized and the L-R stimulus position counterbalanced across trials. A total of 3 replicate trials with each of the six stimulus combinations were given in each session (18 trials), for a total of 108 trials with each stimulus type (18 with each number combination).

\section{Results}

General performance. Overall results are illustrated in Figure 5. Consistent with results in the previous experiments, animals were unable to reliably select the smaller of two candy arrays in order to obtain a larger reward. Performance with candy arrays was again significantly below chance $(M=0.27, \operatorname{SEM}=$ $0.04), t(4)=6.46$, with no improvement over performance levels observed during Experiment 1 . Again, the animals continued to select the larger of the two candy arrays and received the smaller reward.

Overall performance with Arabic numerals in place of candy arrays, however, was significantly better than with candy $(M=0.66, S E M=0.05), t(4)=9.29$, and was now significantly above chance, $t(4)=3.17$. As is illustrated in Figure 5, this performance increment was apparent immediately upon introduction of the numerical stimuli and was stable over all sessions in which numerals served as choice stimuli. These findings indicate that the interference effect arising from an apparent response disposition toward the larger of two arrays can be overcome by the use of number symbols as choice stimuli.

Array size and disparity. Consistent with the results of Experiments 1 and 2, performance with candy arrays was related to the disparity between the two choice arrays. As illustrated in Figure 6A, the disparity ratio again accounted for the majority of the variance in overall performance across the differing numerical combinations of paired-choice arrays $\left(R^{2}=.71\right), F(1,4)=9.57$. The slope of the disparity-ratio function with candy arrays was virtually identical to that obtained in Experiment 1 (see Figures $2 \mathrm{~B}$ and 
6 A). The results again document a remarkably stable pattern of performance with candy arrays across both animals and experiments.

However, as illustrated in Figure 6B, a distinctly different pattern of results was apparent with Arabic numerals as array elements. The more optimal performance observed with numerals was characterized by both (a) a significant increase in the overall mean level of performance (see above) and (b) a significant decrease in the slope of this function (for candies, $M$ slope $=-.48, S E M=0.05$; for numerals, $M$ slope $=-.15, S E M=0.05), t(4)=4.22$. Moreover, in contrast to candy sessions, the slope accounted for only a negligible and nonsignificant proportion of variance in performance with Arabic numerals $\left(R^{2}=\right.$ .25). These findings indicate that performance with numeric symbols was largely devoid of a disparitydependent interference, whereas this interference continued to be potent when candy arrays served as choice stimuli.

Individual differences. As was the case in Experiments 1 and 2, there were minimal individual differences among the animals in performance with candy arrays (all means ranged from .19 to .40). Nonparametric analyses revealed that each animal's performance was significantly below chance, all $\chi^{2} s(1, N=108)>$ 4.48 , and that there was also only moderate variation in the slopes of the disparity-ratio functions $(M=$ $-.48, \mathrm{SEM}=0.05$; range $=-.38$ to -.64 ) across animals.

Overall performance of each animal was significantly higher with Arabic numerals as stimuli, relative to sessions with candy arrays ( $M$ performance increment $=+.39$, range $=+.26$ to +.48 ), all $X^{2} s(1, N=216)>$ 15.1. Associated with this performance increment, each animal evidenced a decrease in the slope of the disparity-ratio-performance function and in the proportion of variance in performance that could be accounted for by the slope. Although correlations were observed between the slope of the disparity-ratio function and overall performance with numerals $(r=.65)$ and between the changes in slope and performance from candies to numerals $(r=.49)$, neither relationship achieved significance.

\section{Discussion}

The present interference paradigm pitted the potential direct response biases arising from the tendency to select the larger of two candy arrays against the response dispositions based on the reward contingency (animals received the nonselected array). In Experiments 1 and 2, the direct response biases associated with array size introduced a sizeable interference with optimal performance defined by the reward outcome. The present results reveal that this performance interference could be substantially attenuated if Arabic numerals were substituted for the candy arrays as choice stimuli. This led to a decrease in the interference slope (disparity-ratio function) and an associated improvement in performance above chance levels. It is important to note that this improved performance was apparent immediately upon introduction of the number stimuli and did not require further training. This finding suggests that the animals had acquired knowledge of the rule structure of the task but were unable to implement this knowledge because of the competing response bias toward the larger array. Thus, the Arabic symbols appeared to capture the requisite numerical information of the stimuli without encompassing the incentive properties that subserved the interfering response bias.

The present results confirm our previous report of the improved performance of a single animal (Sheba) when Arabic numerals were substituted for candy arrays as choice stimuli (Boysen \& Berntson, 1995). In both cases, performance was significantly above chance with Arabic numerals and significantly below chance with candy arrays. Comparison of the animal (Sheba) tested in both studies again revealed a high degree of individual stability in performance. Sheba's overall performance in the corresponding phase of the previous study (Phase 3) was $21 \%$ with candies and $70 \%$ with numerals, compared with $24 \%$ and $72 \%$, respectively, in the present experiment. ${ }^{\S}$ 
Figure 6. Regression functions illustrating the relationship between the probability of optimal response and the disparity ratio (numeric disparity-total size of the arrays) for candy array and Arabic numeral stimulus choices. The heavy line depicts the mean regression function, and the dotted lines illustrate individual regression functions for the 5 subjects. Dots depict the mean data points for each array combination tested.
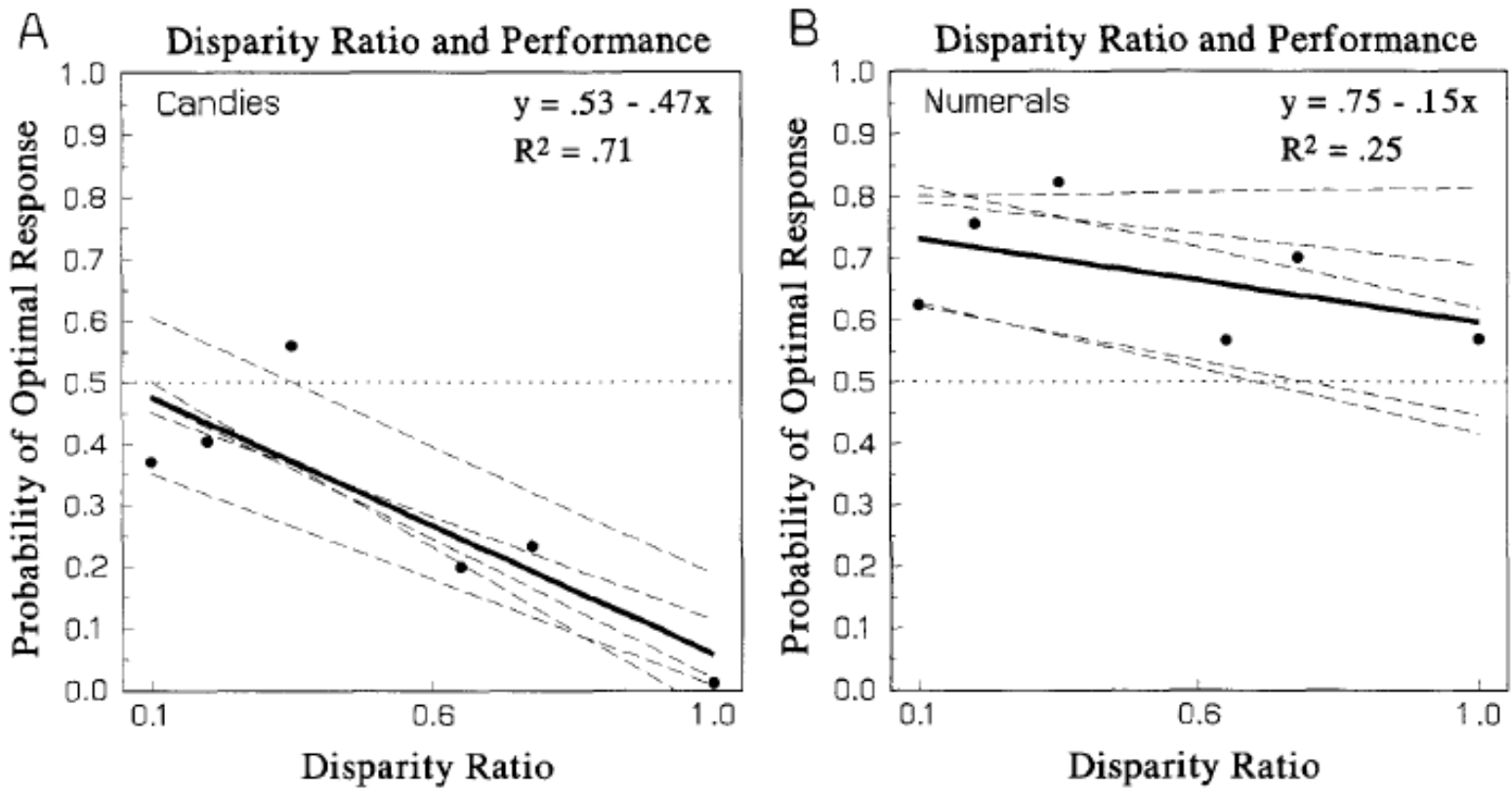

\section{General Discussion}

Results confirm and extend our preliminary observations (Boysen \& Berntson, 1995) of a powerful interference effect in a choice task in which the smaller of two candy arrays must be selected in order to receive the larger (nonselected) array as a reward. None of the 5 animals in the present study was able to demonstrate an optimal response strategy by selecting the smaller candy array, despite hundreds of trials on this task. Rather, animals consistently selected the larger array and, thus, reliably received the smaller reward. The present results further indicate that the poor performance on the task was not attributable to the conspecific food-competition context used in our previous study.

Despite their poor performance, the animals' selections were in fact sensitive to the magnitude of the numerical difference between the choice stimuli and, hence, the reward differential. Increases in disparity among the choice pairs, however, were associated with progressively poorer performance. Performance was, in fact, poorest under the very conditions in which the animal stood to benefit the most by the optimal selection of the smaller array. This raises the possibility that a direct incentive attraction to the larger array may have introduced a competing approach disposition that interfered with optimal task performance. The findings are reminiscent of the self-control literature in animals and children (Eisenberger, Weier, Masterson, \& Theis, 1989; Logue, 1988; Mischel et al., 1989; Tobin \& Logue, 1994). Young children, for example, have difficulty in inhibiting a direct response to food in order to achieve a larger but delayed reward (Mischel et al., 1989). Similarly, adult humans are more impulsive and show poorer self-control when food reinforcers are delivered immediately after a response than when reinforcement is delayed (Forzano \& Logue, 1994). 
Although performance was highly sensitive to the numerical disparity between the array stimuli, the animals did not appear to be making absolute disparity judgments. The effects of disparity were qualified by the overall size of the stimulus arrays, with a given disparity yielding smaller effects with larger overall array sizes. The function that most closely accounted for the animals' performance was not disparity, per se, but the ratio of disparity to the mean array size (which accounted for more than $90 \%$ of the variance in performance with candy arrays). Thus, it appears that the animals' judgments of the differential incentive values of the choice stimuli were relative, consistent with findings in monkeys (Schrier, 1956) and dolphins (Mitchell, Yao, Sherman, \& O'Regan, 1985). These results are also highly reminiscent of the microeconomic "marginal utility" functions of humans, in which the relative effectiveness of a given increment in payoff diminishes as the base size of the payoff is increased (Kreps, 1988).

The apparent interference effect on task performance was largely eliminated when Arabic numerals were substituted for the candy arrays as choice stimuli. Upon introduction of Arabic symbols, performance increased immediately to more optimal levels. This suggests that the animals had in fact acquired implicit knowledge of the rule structure of the task, despite the fact that they were unable to implement this knowledge with candy arrays as stimuli. Indeed, over counterbalanced sessions with symbols and candy arrays, performance shifted immediately from significantly above chance with Arabic symbols to significantly below chance with candy arrays. These findings are again reminiscent of the self-regulation literature, as children who were unable to inhibit suboptimal approach tendencies to food items in the Mischel et al. (1989) study were able to respond more adaptively when the food stimuli were presented symbolically in slide photographs.

The high-incentive items (candies) that composed the stimulus arrays in Experiment 1 likely contributed to the observed interference effect on performance. The overall group performance with rock arrays in Experiment 2 (although significantly higher than that with candies for 2 of the animals) also did not exceed chance levels. The persistence of interference with rock arrays suggests that task interference may not be entirely accounted for by the inherent incentive properties of the array elements. Although the rock stimuli would be expected to have little inherent incentive attraction, the rocks may have accrued incentive value by stimulus generalization, given the perceptual similarities (e.g., in numerosity) between the rock and candy arrays. Alternatively, the rock arrays may have acquired incentive value by association with the candy reinforcers used throughout the study. A perhaps unlikely nonassociative possibility is that, for these animals, bigger is intrinsically better. Although this may be true in an appetitive context, it certainly would not be expected if the stimuli were aversive. Thus, regardless of the specific source of the residual interference effects with rock arrays, this interference appears to be incentive based and associated with the appetitive context of the task.

In contrast to the consistency of performance across animals in candy sessions, there were notable individual differences in performance with rocks. Performance of 2 of the 5 animals was significantly higher with rocks than with candies, albeit significantly lower than with Arabic numerals. These 2 animals (Sarah and Sheba) are distinguished in having had the most extensive training, and being the most accomplished, in symbolic representations. These animals also displayed the highest performance with Arabic symbols in Experiment 3. These findings suggest that the rock arrays, at least for some animals, may minimize the interfering bias toward the larger stimulus array and permit somewhat more optimal performance based on the reinforcement contingency. Even for these 2 animals, however, rock arrays were not nearly as effective as Arabic symbols in minimizing interference.

The present findings may be viewed within the broader framework of evaluative processes (Berntson, Boysen, \& Cacioppo, 1993; Cacioppo \& Berntson, 1994). The evaluative dimension has been widely recognized as a pervasive organizing principle that captures the bivalent, directional component of behavior (Konorski, 1948; Mackintosh, 1989). Evaluative dispositions, characterized by approach- 
avoidance tendencies or appetitive-aversive reactions, are ubiquitous in behavioral contexts. Veridical evaluations of the adaptive significance of environmental objects and events are so central to survival that all species have biological mechanisms for approaching, acquiring, or ingesting certain classes of stimuli; for withdrawing from, avoiding, or rejecting others; and for the establishment of enduring response predispositions toward classes of stimuli.

Of relevance to the present studies is the fact that evaluative organization is apparent across multiple levels of neurobehavioral organization. Manifestations of evaluative dispositions can range from simple pain-withdrawal reflexes to conditioned approach-avoidance responses to generalized attitudinal predispositions toward broad classes of stimuli (Berntson et al., 1993). The significance of this multiplicity of levels in evaluative mechanisms lies in the possibility that multiple evaluative dispositions may be expressed in a given context. Viewed in this framework, two evaluative dispositions may be operative in the present food-selection paradigm: (a) a lower level, nonassociative, inherent approach disposition based on the perceptual-incentive features of the choice stimuli, and (b) a higher, associative disposition based on the reinforcement contingencies.

In natural situations, these dispositions may generally lead to concordant behavioral actions; Menzel and Draper (1965) suggested commonalities between the direct choice responses of primates and selections based on sign learning. In the present study, however, response biases arising from the incentive features of the choice stimuli were directly pitted against the associative disposition based on the reinforcement contingency. Although the animals had clearly acquired the food-distribution rule structure, as evidenced by their performance with Arabic numerals, the direct incentive features of the candy arrays introduced a powerful conflicting disposition. The relative potency of the interfering disposition in the present task is likely related to the perceptual immediacy of the candy arrays, relative to the delay inherent in post-response reinforcement (Forzano \& Logue, 1994; Logue, 1988).

The improved performance with Arabic numbers may be attributable to the fact that these symbolic stimuli failed to evoke the interfering, nonassociative disposition. That is, the Arabic symbols appeared to capture the requisite numerical information of the stimuli, without encompassing the incentive properties that trigger the interfering response bias. Evidence for this view comes from the relatively flat slopes of the disparity-ratio functions with Arabic symbols, in contrast to the negative slopes of the functions with candy arrays. The flat slopes with numerals indicate that, although the animals were making appropriate quantity judgments with Arabic symbols, these judgments were insensitive to the incentive disparity of the reward quantities they represented.

The ability of symbols to encompass selective features or attributes of their real-world referents may constitute an important advantage of symbolic representations. Symbols may permit an organism to efficiently process selected information and adaptively respond on the basis of that knowledge structure while minimizing potential interference from lower level evaluative mechanisms. In this form, symbols may not show equivalence relationships with their referents (Cerutti \& Rumbaugh, 1993; D'Amato, Salmon, Loukas, \& Tomie, 1985; Mclntire, Cleary, \& Thompson, 1987; Sidman \& Tailby, 1982), because symbols may represent only selective features or attributes of these referents. This would violate the requirement of "symmetry" in the relation between the symbol and its referent. Although equivalence relationships can develop between stimuli and associated reinforcers (e.g., Dube, Mcllvane, Mackay, \& Stoddard, 1987), the present animals had extensive experience and training with Arabic numerals in multiple contexts, and the numeric stimuli were not consistently associated with any specific reinforcer. Rather, the relevant dimension across training contexts was the numeric significance of the Arabic symbols. This is similar to the acquisition and application of number symbols by humans and may account for the apparent lack of incentive interference effects or equivalence relationships with Arabic numerals. 
In an earlier approach, Miller (1959) viewed conflict behavior as the net resultant of unitary, opponent approach-avoidance gradients. Although Miller's analyses were elegant, this approach is limited by its focus on interactions (approach-approach, approach-avoidance) within a single evaluative level of organization (Berntson et al., 1993). If multiple levels in evaluative mechanisms are concurrently activated, there also arises the potential for interactions across these evaluative levels. The latter may not be readily reducible to a single gradient. Thus, although fear and pain both entail negative dispositions, they arise from at least partly distinct neural systems, are controlled or triggered by different stimuli, and have differential effects on behavior.

Relatively primitive evaluative dispositions may have notable impact on the expression of more complex behaviors related to higher neurobehavioral processes (e.g., see Timberlake, 1994). The concepts of instinctive drift (Breland \& Breland, 1961), species-specific defense responses (Bolles, 1970; Bolles \& Fanselow, 1980), preparedness and contrapreparedness (Seligman, 1970), and selective associations (LoLordo \& Droungas, 1989) may all reflect the lower dispositional biases that can shape the likelihood of a specific response or association in a given context. Primitive dispositional biases have also been shown to influence attitudes in humans, as task-irrelevant somatic activity associated with approach or withdrawal reflexes can bias preference judgments toward otherwise neutral stimuli (Cacioppo, Priester, \& Berntson, 1993). Like other species, chimpanzees display a wide range of both acquired and inherent evaluative dispositions, some of which may be apparent within $48 \mathrm{hr}$ of birth and manifest in basic perceptual processing (Berntson \& Boysen, 1989; Berntson et al., 1993; Boysen \& Berntson, 1989a). Clearly, a comprehensive understanding of the behavioral manifestations of evaluative processes may require attention to multiple evaluative levels that can be concurrently expressed and interact in complex ways.

In summary, multiple evaluative dispositions may be expressed in a given behavioral context. Generally, these concurrent dispositions may serve synergistically to promote adaptive responding, although in other cases they may yield cognitive-behavioral conflicts. The nature and behavioral manifestations of these conflicts may offer important insights into the cognitive and evaluative structures of humans and other animals. In view of the potential adaptive significance of conflicts between primitive dispositions and those arising from higher cognitive mechanisms, the emergence of the capacity for symbolic representations may well reflect an evolutionary strategy for resolving such conflicts.

${ }^{\dagger}$ We were primarily interested in the slopes of these functions as an index of the effects of array disparity on performance. The $y$ intercepts are not particularly meaningful, as they reflect an extrapolation of the functions (to a condition of no disparity) rather than actual empirical data points. These values are also quite unstable, being highly sensitive to slight shifts in slope.

‡ The rocks and candies were readily discriminable by color, shape, texture, and size (rocks were approximately 1 in. in diameter, which was almost twice the size of the candies). Hence, little perceptual confusion of rocks and candies would be expected.

$\S$ One apparent difference between the present results and our earlier preliminary study (Boysen \& Berntson, 1995) was the positive slope between numeric disparity and performance with numerals in the earlier study (Figure 1). This contrasts with the essentially null slope of the disparity-ratio performance function with numerals in the present experiment. This difference may be more apparent than real, however. Reexamination of the earlier data revealed that, although the slope was positive, it did not significantly differ from zero. Moreover, in the prior study we illustrated only disparities of 1,2 , and 3 , because larger array disparities can be achieved only by a diminishing combination of array sizes. 
Consequently, only a few trials with larger array disparities were available in that study. When data for the higher disparities is included, however, the slope of the disparity-ratio function for numerals flattens considerably (slope $=0.05$ ).

\section{References}

Berntson, G. G., \& Boysen, S. T. (1989). Specificity of the cardiac response to conspecific vocalizations in chimpanzees (Pan troglodytes). Behavioral Neuroscience, 103, 235-245.

Berntson, G. G., Boysen, S. T., \& Cacioppo, J. T. (1993). Neurobehavioral organization and the cardinal principle of evaluative bivalence. Annals of the New York Academy of Science, 702, 75-102.

Bolles, R. C. (1970). Species-specific defense reactions and avoidance learning. Psychological Review, 77, 32-48.

Bolles, R. C., \& Fanselow, M. S. (1980). A perceptual-defensive-recuperative model of fear and pain. Behavioral and Brain Sciences, 3, 291-301.

Boysen, S. T. (1993). Counting in chimpanzees: Nonhuman principles and emergent properties of number. In S. T. Boysen \& E. J. Capaldi (Eds.), The development of numerical competence: Animal and human models (pp. 39-59). Hillsdale, NJ: Erlbaum.

Boysen, S. T., \& Berntson, G. G. (1989a). Conspecific recognition in the chimpanzee: Cardiac indices of significant others. Journal of Comparative Psychology, 103, 215-220.

Boysen, S. T., \& Berntson, G. G. (1989b). Numerical competence in a chimpanzee (Pan troglodytes). Journal of Comparative Psychology, 103, 23-31.

Boysen, S. T., \& Berntson, G. G. (1995). Responses to quantity: Perceptual vs. cognitive mechanisms in chimpanzees (Pan troglodytes). Journal of Experimental Psychology: Animal Behavior Processes, 21, 83-86.

Boysen, S. T., Berntson, G. G., Shreyer, T. A., \& Hannan, M. B. (1995). Indicating acts during counting by a chimpanzee (Pan troglodytes). Journal of Comparative Psychology, 109, 47-51.

Boysen, S. T., Berntson, G. G., Shreyer, T. A., \& Quigley, K. S. (1993). Processing of ordinality and transitivity by chimpanzees (Pan troglodytes). Journal of Comparative Psychology, 107, 208-215.

Breland, K., \& Breland, M. (1961). The misbehavior of organisms. American Psychologist, 16, 267-269.

Cacioppo, J. T., \& Berntson, G. G. (1994). Relationship between attitudes and evaluative space: A critical review with emphasis on the separability of positive and negative substrates. Psychological Bulletin, 115, 401-423.

Cacioppo, J. T., Priester, J. R., \& Berntson, G. G. (1993). Rudimentary determinants of attitudes: II. Arm flexion and extension have differential effects on attitudes. Journal of Personality and Social Psychology, 65, 5-17.

Cerutti, D. T., \& Rumbaugh, D. M. (1993). Stimulus relations in comparative primate perspective. Psychological Record, 43, 811-821.

D'Amato, M. R., Salmon, D. P., Loukas, E., \& Tomie, A. (1985). Symmetry and transitivity of conditional relations in monkeys (Cebus apella) and pigeons (Columba livid). Journal of the Experimental Analysis of Behavior, 44, 35-47.

Dube, W. V., Mcllvane, W. J., Mackay, H. A., \& Stoddard, L. T. (1987). Stimulus class membership established via stimulus-reinforcer relations. Journal of the Experimental Analysis of Behavior, 47, 159-175.

Eisenberger, R., Weier, P., Masterson, F. A., \& Theis, L. Y. (1989). Fixed-ratio schedules increase generalized self-control: Preference for large rewards despite high effort or punishment. Journal of Experimental Psychology: Animal Behavior Processes, 15, 383-392. 
Forzano, L. B., \& Logue, A. W. (1994). Self-control in adult humans: Comparison of qualitatively different reinforcers. Learning \& Motivation, 25, 65-82.

Fujita, K., \& Matsuzawa, T. (1986). A new procedure to study the perceptual world of animals with sensory reinforcement: Recognition of humans by a chimpanzee. Primates, 27, 283-291.

Gillan, D. J., Premack, D., \& Woodruff, G. (1981). Reasoning in the chimpanzee: I. Analogical reasoning. Journal of Experimental Psychology: Animal Behavior Processes, 7, 1-17.

Hays, W. L. (1988). Statistics. New York: Holt, Reinhart \& Winston.

Konorski, J. (1948). Conditioned reflexes and neuron organization. Cambridge, England: Cambridge University Press.

Kreps, D. M. (1988). Notes on the theory of choice. Boulder, CO: Westview Press.

Logue, A. W. (1988). Research on self-control: An integrating framework. Behavioral and Brain Sciences, $11,665-709$.

LoLordo, V. M., \& Droungas, A. (1989). Selective associations and adaptive specializations: Taste aversions and phobias. In S. Klein \& R. Mowrer (Eds.), Contemporary learning theories: Instrumental conditioning theory and the impact of biological constraints on learning (pp. 145179). Hillsdale, NJ: Erlbaum.

Mackintosh, N. J. (1989). The psychology of animal learning. London: Academic Press.

Matsuzawa, T. (1985). Use of numbers by a chimpanzee. Nature, 315, 57-59.

McIntire, K. D., Cleary, J. P., \& Thompson, T. (1987). Conditional relations by monkeys: Reflexivity, symmetry, and transitivity. Journal of the Experimental Analysis of Behavior, 47, 279-285.

Menzel, E. W., \& Draper, W. A. (1965). Primate selection of food by size: Visible versus invisible rewards. Journal of Comparative and Physiological Psychology, 59, 231-239.

Miller, N. E. (1959). Liberalization of basic S-R concepts: Extensions to conflict behavior, motivation and social learning. In S. Koch (Ed.), Psychology: A study of a science (Study I, Vol. 2, pp. 198-292). New York: McGraw-Hill.

Mischel, W., Shoda, Y., \& Rodriguez, M. L. (1989). Delay of gratification in children. Science, 244, 933937.

Mitchell, R. W., Yao, P., Sherman, P. T., \& O'Regan, M. (1985). Discriminative responding of a Dolphin (Tursiops truncatus) to differentially rewarded stimuli. Journal of Comparative Psychology, 99, 218-225.

Premack, D. (1986). Cavagai. London: Cambridge University Press.

Savage-Rumbaugh, E. S. (1986). Ape language: From conditioned response to symbol. New York: Columbia University Press.

Schrier, A. M. (1956). Amount of incentive and performance on a black-white discrimination problem. Journal of Comparative and Physiological Psychology, 43, 123-125.

Seligman, M. E. P. (1970). On the generality of the laws of learning. Psychological Review, 77, 406-418.

Sidman, M., \& Tailby, W. (1982). Conditional discrimination vs. matching-to-sample: An expansion of the testing paradigm. Journal of the Experimental Analysis of Behavior, 37, 5-22.

Timberlake, W. (1994). Behavioral systems, associationism, and Pavlovian conditioning. Psychonomic Bulletin \& Review, 1. 405-420.

Tobin, H., \& Logue, A. W. (1994). Self-control across species (Columba livia, Homo sapiens, and Rattus norvegicus). Journal of Comparative Psychology, 108, 126-133. 